\title{
Inhaled antibiotics for stable non-cystic fibrosis bronchiectasis: a systematic review
}

\author{
Alessandra Monteiro Brodt ${ }^{1}$, Elizabeth Stovold ${ }^{2}$ and Linjie Zhang ${ }^{1}$
}

Affiliations:

${ }^{1}$ Faculty of Medicine, Federal University of Rio Grande, Rio Grande, Brazil.

${ }^{2}$ Cochrane Airway Group, Population Health Sciences and Education, St George's University of London, London, UK.

\section{Correspondence:}

Linjie Zhang, Faculty of Medicine, Federal University of Rio Grande, Rua Visconde de Paranagua 102, Centro, Rio Grande-RS, Brazil.

E-mail: zhanglinjie63dayahoo.com.br

ABSTRACT We conducted a meta-analysis of randomised trials to evaluate the efficacy and safety of inhaled antibiotics in patients with stable non-cystic fibrosis (CF) bronchiectasis.

We searched the Cochrane Airways Group Register of Trials from inception until March 2014.

12 trials with 1264 adult patients were included, of which five were unpublished studies. Eight trials on 590 patients contributed data to the meta-analysis. Amikacin, aztreonam, ciprofloxacin, gentamicin, colistin or tobramycin were used for 4 weeks to 12 months. Inhaled antibiotics were more effective than placebo or symptomatic treatment in reducing sputum bacterial load (five trials; weighted mean difference $-2.65 \log _{10} \mathrm{CFU} \cdot \mathrm{g}^{-1}, 95 \% \mathrm{CI}-4.38--0.92 \log _{10} \mathrm{CFU} \cdot \mathrm{g}^{-1}$ ), eradicating the bacteria from sputum (six trials; risk ratio 4.2, 95\% CI 1.66-10.64) and reducing the risk of acute exacerbations (five trials; risk ratio 0.72, $95 \%$ CI $0.55-0.94)$. Bronchospasm occurred in $10 \%$ of patients treated with inhaled antibiotics compared with $2.3 \%$ in the control group (seven trials; risk ratio $2.96,95 \%$ CI $1.30-6.73$ ), but the two groups had the same withdrawal rate due to adverse events (12.2\%).

Inhaled antibiotics may provide an effective suppressive antibiotic therapy with an acceptable safety profile in adult patients with stable non-CF bronchiectasis and chronic bronchial infection.

@ERSpublications

Inhaled antibiotics are effective with an acceptable safety profile in adults with stable non-CF bronchiectasis http://ow.ly/wxsGg 


\section{Introduction}

Non-cystic fibrosis (CF) bronchiectasis remains an important cause of chronic respiratory morbidity with a considerable healthcare burden in both developed and developing countries [1-3]. Most patients with bronchiectasis are chronically infected with a variety of bacterial pathogens, resulting in a vicious cycle of infection and inflammation with persistent respiratory symptoms and further airway damage [4, 5]. A recent study found a direct relationship between bacterial load and airway and systemic inflammation in 385 patients with stable non-CF bronchiectasis [6]. Higher bacterial load was associated with more severe respiratory symptoms, greater risk of exacerbations and more frequent unscheduled hospitalisations. This study also showed that both 14-day intravenous antibiotic therapy and 12-month treatment with nebulised gentamicin had significantly reduced markers of airways and systemic inflammation. In contrast, a Cochrane systematic review of nine randomised trials involving 378 patients with non-CF bronchiectasis reported no benefit of prolonged ( $\geqslant 4$ weeks) antibiotics in reducing risk of exacerbations (OR 0.96, 95\% CI 0.27-3.45), in spite of a higher clinical response rate (OR 3.37, 95\% CI 1.6-7.09) [7]. The review included three trials in which nebulised antibiotics were used, but the therapeutic benefit of this group of antibiotics was not separately assessed. Given the potential advantage of inhalation over other routes of administration for antibiotics, use of inhaled antibiotics in patients with non-CF bronchiectasis needs to be addressed. We conducted this systematic review and meta-analysis of randomised trials to assess the efficacy and safety of inhaled antibiotics in patients with stable non-CF bronchiectasis.

\section{Methods}

We followed the recommendations of the PRISMA (Preferred Reporting Items for Systematic Reviews and Meta-Analyses) statement [8] for conduct and reporting of this review. The review protocol was completed in 2011 and approved by a panel of experts consisting of one epidemiologist and two pulmonologists. The only difference between the protocol and review is that we included trials involving patients with stable disease treated with inhaled antibiotics for $\geqslant 4$ weeks, rather than patients in any clinical situation with any treatment duration as initially described in the review protocol.

\section{Data sources and search strategy}

We searched the Cochrane Airways Group Register of Trials, which contains trials identified through the Cochrane Central Register of Controlled Trials, MEDLINE, EMBASE, CINAHL, LILACS, AMED and PsycINFO, and undertook hand searching of respiratory journals and meeting abstracts. The search strategy used was as follows: (( $\left(\right.$ antibiotic $^{\star}$ or antibacterial or anti-bacterial or antimicrobial or anti-microbial or $\left.\operatorname{aminoglycoside}^{\star}\right)$ and $\left(\right.$ inhal $^{\star}$ or nebuli* or aerosol $\left.{ }^{\star}\right)$ ) or tobramycin or gentamicin or colomycin or aztreonam or taurolidine or cephaloridine or carbenicillin or ceftazidime or colistin or amikacin or vancomycin) AND (bronchiectasis or chronic bronchial infection or chronic bronchial sepsis). We also conducted a search of the ClinicalTrials.gov trials registry. All databases were initially searched from their inception until November 2011, and update searches were conducted in November 2013 and March 2014. There was no restriction on language of publication. We checked reference lists of all primary studies and review articles for additional relevant trials.

\section{Study selection}

Inclusion and exclusion criteria were defined a priori. To be included in this review, studies had to meet all of the following criteria. 1) Study design: a randomised controlled trial. 2) Participants: adult or paediatric patients with bronchiectasis diagnosed by bronchography or computed tomography, and in a stable clinical condition. 3) Interventions and comparisons: antibiotics administered via inhalation for $\geqslant 4$ weeks, compared with placebo or other interventions. 4) Outcomes (at least one of the following measures was obtained): sputum analysis (microbiological examination and/or physical properties), clinical response, acute exacerbations, hospital admission, pulmonary function test, health-related quality of life, markers of airways and systemic inflammation, and safety. Trials were excluded if patients had a diagnosis of CF or other chronic pulmonary disorders, or had an acute exacerbation at study entry.

Two investigators independently screened the titles and abstracts of citations identified by the searches. Full articles were retrieved when they appeared to meet the inclusion criteria or there were insufficient data in the titles and abstracts to make a clear decision for their inclusion. The definitive inclusion of trials was made after reviewing the full text of the articles. Any disagreement between the two reviewers about study inclusion was resolved by discussion.

\section{Data extraction and assessment of risk of bias in included studies}

Data from each included study were extracted by one reviewer and confirmed by another. We used a predesigned standard form to extract five categories of data. 1) General information: first author's name, year 
of publication, country of origin and funding source. 2) Methodology of the study: study design, methods of random sequence generation, allocation concealment and blinding, description of withdrawal, methods of collection and reporting of efficacy and safety outcomes and adherence with treatment. 3) Participants: age, sex, number enrolled, randomised and analysed, inclusion and exclusion criteria, method for diagnosis of bronchiectasis and baseline characteristics of patients. 4) Interventions and comparisons: specific antibiotic, placebo or other comparator, daily dose, interval of administration, duration of treatment, drug delivery device, and co-interventions. 5) Outcomes: for continuous outcomes, we extracted sample size, mean (median) and precision of measurements (standard deviation, standard error, 95\% confidence intervals or interquartile range (IQR)) for each treatment arm. For dichotomous outcomes, we extracted number of events and total number of participants in each treatment arm. Intention-to-treat (ITT) datasets were used whenever available. In one trial [9], we used Engauge digitising software (digitizer.sourceforge.

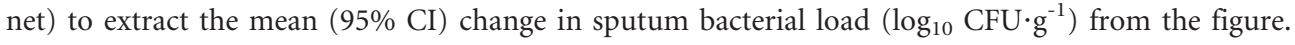

Two reviewers independently assessed the risk of bias in included trials by examining six key domains according to the recommendations of the Cochrane Collaboration [10]: 1) allocation sequence generation; 2) concealment of allocation; 3) blinding of participants and investigators; 4) incomplete outcome data; 5) selective outcome reporting; and 6) other sources of bias. We graded each potential source of bias as "yes", "no" or "unclear", relating to whether the potential for bias was high, low or unknown. Any disagreement about data extraction and risk of bias assessment was resolved by discussion.

\section{Data synthesis}

We performed meta-analysis for quantitative data synthesis whenever there were available data from the primary studies. For continuous outcomes, weighted mean difference (WMD) between treatment groups and $95 \%$ confidence intervals were used as the metrics of effect size. Dichotomous data were synthesised using risk ratios and $95 \%$ confidence intervals as the effect measures. We also calculated number needed to treat $(\mathrm{NNT})$ and number needed to harm $(\mathrm{NNH})$ to measure the effect size of benefits and harms of the treatment. We used the random-effects model for meta-analysis because it is more appropriate than the fixed-effects model and gives more conservative estimates with wider confidence intervals when there is significant heterogeneity across studies. Otherwise, the two models generate similar results. We used the $\mathrm{I}^{2}$ statistic to measure heterogeneity among the trials in each analysis. The $\mathrm{I}^{2}$ statistic ranges from $0 \%$ to $100 \%$ and measures the degree of inconsistency of results across studies, with values of $25 \%, 50 \%$ and $75 \%$ corresponding to low, moderate and high heterogeneity, respectively [10]. We conducted planned subgroup analyses to explore the potential sources of heterogeneity and effect modifiers. In one trial [11], we obtained the missing standard deviation of the mean change in sputum bacterial load $\left(\log _{10} \mathrm{CFU} \cdot \mathrm{g}^{-1}\right)$ from study results posted in the ClinicalTrials.gov trials registry. The statistical analysis was performed with Stata version 11.0 (StataCorp, College Station, TX, USA).

\section{Results}

Search results and study selection

The search strategy identified 126 unique records. After screening the titles and abstracts, we retrieved 17 potentially relevant full-text articles for further evaluation. Nine articles [6, 12-19] were excluded for reasons shown in figure 1. The findings of one trial were reported by BARKER et al. [9] in 2000 and by COUCH [20] in 2001, so this trial was labelled as BARKer 2000 and COUCH 2001. We obtained data from the trials registry (ClinicalTrials.gov), congress presentations and drug company's trial reports to assess the eligibility of five unpublished trials. All five unpublished trials [21-28] met the inclusion criteria. No additional trials were found by checking the reference lists of primary studies and review articles. Thus, 12 trials $[9,11,21-33]$ were included in this review with eight $[9,11,26,27,29-33]$ contributing data to the meta-analysis (fig. 1).

\section{Study characteristics and risk of bias assessment}

Table 1 summarises the characteristics of the 12 included trials. All but three studies [28, 31, 32] were randomised, double-blind, placebo-controlled trials and nine $[9,11,21-27,30,31,33]$ were multicentre studies. Eight trials [9, 11, 21-27, 31, 33] were sponsored by pharmaceutical companies. The 12 trials included a total of 1264 adult patients with clinically stable computed tomography-confirmed non-CF bronchiectasis and chronic bronchial infection; 656 were allocated to the antibiotic group and 608 were allocated to the control group. None of the trials included paediatric patients. Eight trials with a total of 590 patients (304 in the antibiotic group and 286 in the control group) contributed data to the meta-analysis. In the remaining four unpublished trials with a total of 674 patients, we failed to obtain suitable data for metaanalysis. We have narratively summarised the main findings of these four trials in online supplementary table S1 [21-25, 28]. 
FIGURE 1 Flow diagram of study selection. CAGR: Cochrane Airways Group Register of Trials.

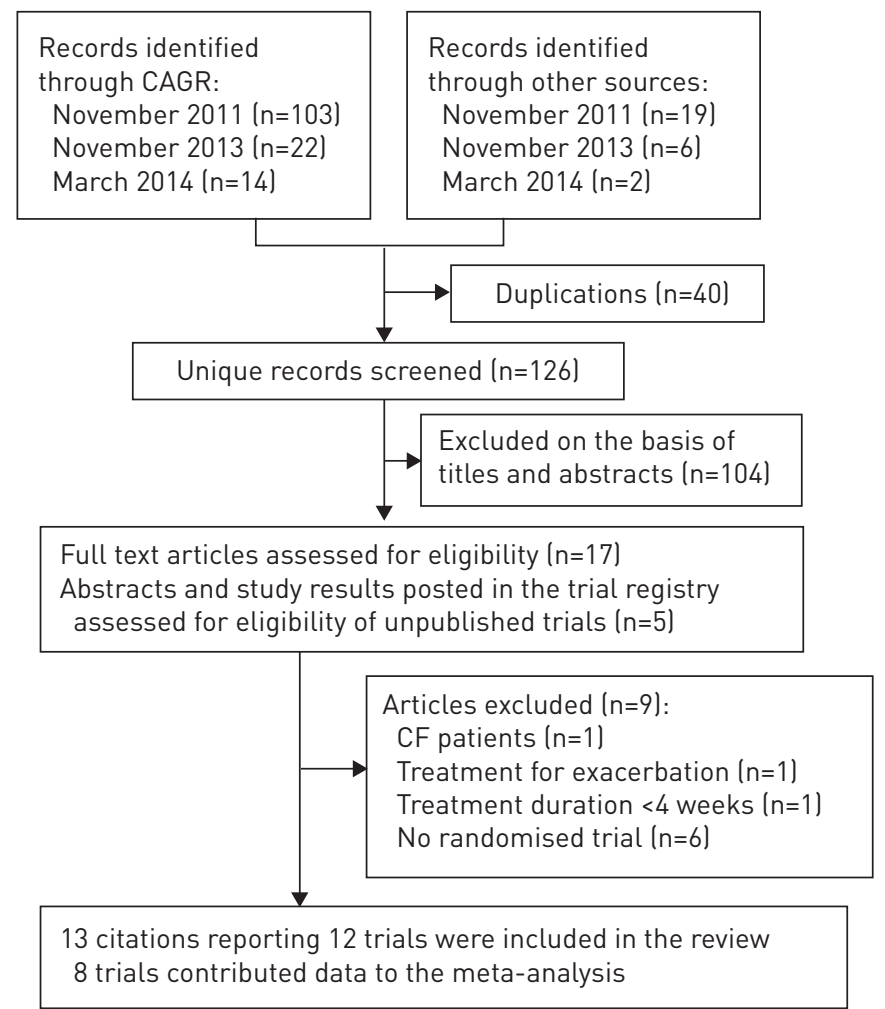

The inhaled antibiotics used in the 12 trials were amikacin [26, 27], aztreonam [21-23] ciprofloxacin $[11,24,25,33]$, colistin $[26,27,30]$, gentamicin [31] and tobramycin [9, 29, 32]. A jet nebuliser was used as the inhalation device in all but one trial [11], in which a dry-powder inhaler was used for delivery of ciprofloxacin. The treatment duration varied from 4 weeks to 12 months.

All but one [32] of the 12 trials clearly defined primary and secondary outcomes. The primary efficacy

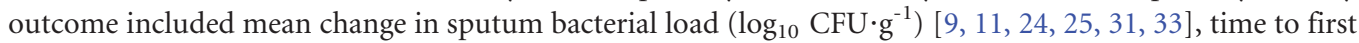
exacerbation or number of exacerbations [29, 30], mean change in quality of life scores [21-23] and need for hospital readmissions [28]. One safety trial [26, 27] used incidence of adverse events as the primary outcome. All but one [30] of the eight trials included in the meta-analysis used per-protocol population for the analysis of efficacy outcomes. Safety outcomes were analysed in all trials using ITT population.

The risk of bias of the eight trials which contributed data to the meta-analysis was assessed (online supplementary table S2). All but one trial [33] had an unknown risk of selection bias due to lack of information on random sequence generation and allocation concealment. Attribution bias might have occurred in all but one trial $[26,27]$, because of high and unbalanced withdrawal rates after randomisation in the treatment groups.

\section{Efficacy of inhaled antibiotics \\ Sputum microbiology}

Seven trials with a total of 608 patients [9, 11, 26, 27, 29-31, 33] reported mean change from baseline in

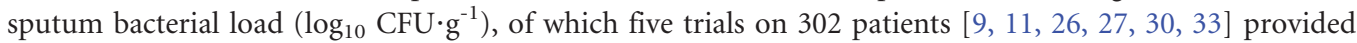
suitable data for pooling of results. One 6-month trial [30] reported mean change from baseline in sputum Pseudomonas aeruginosa load at 4 and 12 weeks, but not at the end of the 6-month treatment. The mean $\pm \mathrm{SD}$ reduction of $P$. aeruginosa load in the colistin versus placebo groups was $-1.7 \pm 2.2$ versus $-0.3 \pm 1.9 \log _{10} \mathrm{CFU} \cdot \mathrm{g}^{-1}$ and $-1.6 \pm 2.2$ versus $-0.5 \pm 2.3 \log _{10} \mathrm{CFU} \cdot \mathrm{g}^{-1}$ at 4 and 12 weeks, respectively. We used the results obtained at 4 weeks for the meta-analysis because the other four trials had 4-week treatment duration. The meta-analysis of the five trials showed that inhaled antibiotics produced a greater reduction in sputum bacterial load $\left(\log _{10} \mathrm{CFU} \cdot \mathrm{g}^{-1}\right)$ compared with the control group (WMD $-2.65,95 \% \mathrm{CI}$ $-4.38--0.92 ; \mathrm{p}=0.003, \mathrm{I}^{2}=95.2 \%$ ) (fig. 2 ). Subgroup analysis showed a statistically significant difference in reducing sputum bacterial $\operatorname{load}\left(\log _{10} \mathrm{CFU} \cdot \mathrm{g}^{-1}\right)$ between different types of antibiotics (aminoglycosides, two trials with 123 patients, WMD -2.45, 95\% CI -6.55-1.64; colistin, one trial on 144 patients, WMD -1.40, 


\section{TABLE 1 Characteristics of included trials}

\begin{tabular}{|c|c|c|c|c|c|c|}
\hline Study & Country & Participants & Intervention & Control & Study design & Outcomes \\
\hline $\begin{array}{l}\text { AIR-BX1 and } \\
\text { AIR-BX2 trials } \\
2013 \text {, } \\
\text { sponsored by } \\
\text { Gilead } \\
\text { Sciences } \\
\text { [21, 22, 23] }\end{array}$ & $\begin{array}{l}\text { Belgium, } \\
\text { France, } \\
\text { Germany, } \\
\text { Italy, the } \\
\text { Netherlands, } \\
\text { Spain, UK, USA }\end{array}$ & $\begin{array}{l}\text { Inclusion criteria: adult patients ( } \geqslant 18 \text { years) had non-CF } \\
\text { bronchiectasis confirmed by HRCT, and had positive } \\
\text { sputum culture for Gram-negative organisms } \\
\text { Exclusion criteria: patients had hospitalisation within } \\
14 \text { days prior to the study, previous exposure to inhaled } \\
\text { aztreonam, continuous } \mathrm{O}_{2} \text { use }>2 \mathrm{~L} \cdot \mathrm{min}^{-1} \text {, current } \\
\text { treatment for non-TB mycobacterial infection or active } \\
\text { TB infection within } 1 \text { year of enrolment or other serious } \\
\text { medical conditions }\end{array}$ & $\begin{array}{l}\text { Aztreonam } \\
75 \mathrm{mg} \text { via an } \\
\text { eFlow nebuliser, } \\
\text { three times a day, } \\
\text { two 28-day } \\
\text { on/off cycles } \\
\text { AIR-BX1 ( } \mathrm{n}=134) \\
\text { AIR-BX2 }(\mathrm{n}=136)\end{array}$ & $\begin{array}{l}\text { Placebo via an } \\
\text { eFlow nebuliser, } \\
\text { three times a } \\
\text { day, two } 28 \text {-day } \\
\text { on/off cycles } \\
\text { AIR-BX1 } \\
\text { (n=132) } \\
\text { AIR-BX2 } \\
(n=138)\end{array}$ & $\begin{array}{l}\text { International, } \\
\text { multicentre, } \\
\text { RDBPCT }\end{array}$ & $\begin{array}{l}\text { Primary: mean change in QoL-B } \\
\text { scores from baseline to day } 28 \\
\text { Secondary: mean change in } \\
\text { QoL-B scores from baseline to } \\
\text { day 84, time to first } \\
\text { exacerbation }\end{array}$ \\
\hline $\begin{array}{l}\text { BARKER } 2000 \text { and } \\
\text { COUCH } 2001 \text {, } \\
\text { sponsored by } \\
\text { PathoGenesis } \\
{[9,20]}\end{array}$ & USA & $\begin{array}{l}\text { Inclusion criteria: adult patients ( } \geqslant 18 \text { years) had } \\
\text { bronchiectasis diagnosed by } \mathrm{CT} \text {, and had grossly } \\
\text { purulent sputum containing PA } \geqslant 10^{4} \mathrm{CFU} \cdot \mathrm{g}^{-1} \\
\text { Exclusion criteria: patients had CF, ABPA, acute pulmonary } \\
\text { process requiring medical intervention, or had received } \\
\text { antibiotics within } 2 \text { weeks prior to the study }\end{array}$ & $\begin{array}{l}\text { Tobramycin } \\
300 \mathrm{mg} \text { via a jet } \\
\text { nebuliser, twice } \\
\text { daily, } 4 \text { weeks } \\
\quad[\mathrm{n}=37]\end{array}$ & $\begin{array}{l}\text { Placebo } \\
\text { (1.25 mg quinine } \\
\text { sulfate) via a jet } \\
\text { nebuliser, twice } \\
\text { daily, } 4 \text { weeks } \\
\text { (n }=37)\end{array}$ & $\begin{array}{l}\text { Multicentre, } \\
\text { RDBPCT }\end{array}$ & $\begin{array}{l}\text { Primary: mean change in sputum } \\
\text { PA density }\left(\log _{10} \mathrm{CFU} \cdot \mathrm{g}^{-1}\right) \\
\text { from baseline to week } 4 \\
\text { Secondary: eradication of PA in } \\
\text { sputum, clinical improvement, } \\
\text { hospitalisation, } \mathrm{PFT} \text {, adverse } \\
\text { events, emergency of bacterial } \\
\text { resistance }\end{array}$ \\
\hline $\begin{array}{c}\text { DroBnic 2005, no } \\
\text { sponsor [29] }\end{array}$ & Spain & $\begin{array}{l}\text { Inclusion criteria: adult patients had bronchiectasis } \\
\text { diagnosed by HRCT, and had } \geqslant 3 \text { positive sputum } \\
\text { cultures for PA during the } 6 \text { months before the study } \\
\text { Exclusion criteria: patients had } \mathrm{CF} \text {, tobramycin } \\
\text { hypersensitivity, PA in sputum resistant to tobramycin, } \\
\text { or had serum creatinine } \geqslant 1.5 \mathrm{mg} \cdot \mathrm{dL}^{-1}\end{array}$ & $\begin{array}{l}\text { Tobramycin } \\
300 \text { mg via a jet } \\
\text { nebuliser, twice } \\
\text { daily, } 6 \text { months } \\
\quad(n=30)\end{array}$ & $\begin{array}{l}\text { Placebo }(0.9 \% \\
\text { saline) via a jet } \\
\text { nebuliser, twice } \\
\text { daily, } 6 \text { months } \\
\quad(n=30)\end{array}$ & $\begin{array}{l}\text { Crossover } \\
\text { RDBPCT, with } \\
\text { a one-month } \\
\text { washout } \\
\text { period }\end{array}$ & $\begin{array}{l}\text { Primary: number of exacerbations } \\
\text { and hospitalisations } \\
\text { Secondary: mean change in } \\
\text { sputum PA density, } \\
\text { eradication of PA in sputum, } \\
\text { use of antibiotic, PFT, markers } \\
\text { of systemic inflammation, } \\
\text { SGRQ, adverse events, } \\
\text { emergence of bacterial } \\
\text { resistance }\end{array}$ \\
\hline $\begin{array}{l}\text { HAWORTH 2014, } \\
\text { sponsored by } \\
\text { Profile } \\
\text { Pharma Ltd } \\
\text { [30] }\end{array}$ & $\begin{array}{c}\text { Russia, } \\
\text { Ukraine, UK }\end{array}$ & $\begin{array}{l}\text { Inclusion criteria: adult patients ( } \geqslant 18 \text { years) had clinically } \\
\text { stable, non-CF bronchiectasis conformed by } C T \text {, had } \geqslant 2 \\
\text { positive sputum cultures for PA in the preceding } \\
12 \text { months and a positive sputum culture for PA at the } \\
\text { screening visit } \\
\text { Exclusion criteria: not stated }\end{array}$ & $\begin{array}{l}\text { Colistin } \\
1 \text { million IU via } \\
\text { I-neb AAD } \\
\text { system, twice } \\
\text { daily, } 6 \text { months } \\
\text { (n=73) }\end{array}$ & $\begin{array}{l}\text { Placebo }(0.45 \% \\
\text { saline) via I-neb } \\
\text { AAD system, } \\
\text { twice daily, } \\
6 \text { months } \\
\text { (n=71) }\end{array}$ & $\begin{array}{l}\text { Multicentre } \\
\text { RDBPCT }\end{array}$ & $\begin{array}{l}\text { Primary: time to first } \\
\text { exacerbation } \\
\text { Secondary: severity of } \\
\text { exacerbation, mean change in } \\
\text { sputum } P A \text { density } \\
\left(\log _{10} \mathrm{CFU} \cdot \mathrm{g}^{-1}\right), \mathrm{SGRQ} \text {, } \\
\text { adverse events, emergence of } \\
\text { bacterial resistance }\end{array}$ \\
\hline $\begin{array}{l}\text { MURRAY 2011, } \\
\text { supported by } \\
\text { the Chief } \\
\text { Scientist } \\
\text { Office, } \\
\text { Scotland [31] }\end{array}$ & UK & $\begin{array}{l}\text { Inclusion criteria: adult patients had clinically stable } \\
\text { bronchiectasis diagnosed by HRCT, had chronically } \\
\text { infected sputum, had } \geqslant 2 \text { exacerbations in the past year, } \\
\text { and had FEV }>30 \% \text { predicted } \\
\text { Exclusion criteria: patients had CF, active pulmonary } \\
\text { disorders (TB, sarcoidosis, ABPA), COPD, poorly } \\
\text { controlled asthma, creatinine clearance }<30 \mathrm{~mL} \cdot \mathrm{min}^{-1} \text {, } \\
\text { vestibular instability, or had hypersensitivity to } \\
\text { aminoglycosides }\end{array}$ & $\begin{array}{l}\text { Gentamicin } \\
80 \mathrm{mg} \text { via a jet } \\
\text { nebuliser, twice } \\
\text { daily, } 12 \text { months } \\
\quad(\mathrm{n}=32)\end{array}$ & $\begin{array}{l}\text { Placebo }(0.9 \% \\
\text { saline) via a jet } \\
\text { nebuliser, twice } \\
\text { daily, } 12 \text { months } \\
\quad(n=33)\end{array}$ & $\begin{array}{l}\text { Multicentre, } \\
\text { randomised, } \\
\text { single-blind, } \\
\text { placebo- } \\
\text { controlled trial }\end{array}$ & $\begin{array}{l}\text { Primary: mean change in sputum } \\
\left.\text { bacterial load (log }{ }_{10} \mathrm{CFU} \cdot \mathrm{g}^{-1}\right) \\
\text { Secondary: sputum analysis } \\
\text { (volume, purulence, MPO, free } \\
\text { elastase, bacterial eradication), } \\
\text { acute exacerbation, PFT, SGRQ, } \\
\text { LCQ, markers of systemic } \\
\text { inflammation, adverse events, } \\
\text { emergence of bacterial } \\
\text { resistance }\end{array}$ \\
\hline $\begin{array}{l}\text { ORBIT-1 } \\
\text { trial 2011, } \\
\text { sponsored by } \\
\text { Aradigm Corp. } \\
{[24,25]}\end{array}$ & $\begin{array}{c}\text { Australia, } \\
\text { Canada, } \\
\text { Germany, UK, } \\
\text { USA }\end{array}$ & $\begin{array}{l}\text { Inclusion criteria: adult patients ( } \geqslant 18 \text { years) had clinically } \\
\text { stable, non-CF bronchiectasis confirmed by HRCT, and } \\
\text { had PA airway infection } \\
\text { Exclusion criteria: patients had either changes in regimen } \\
\text { or initiation of azithromycin, hypertonic saline, } \\
\text { bronchodilators or oral steroids within } 28 \text { days prior to } \\
\text { study, had received investigational drug or device within } \\
28 \text { days prior to study, or had any serious or active } \\
\text { medical or psychical conditions }\end{array}$ & $\begin{array}{c}\text { Liposomal } \\
\text { ciprofloxacin } \\
100 \mathrm{mg}(\mathrm{n}=30) \text {, } \\
200 \mathrm{mg}(\mathrm{n}=32) \\
\text { via a nebuliser, } \\
\text { once daily, } \\
28 \text { days }\end{array}$ & $\begin{array}{l}\text { Matching } \\
\text { placebo via a } \\
\text { nebuliser, once } \\
\text { daily, } 28 \text { days } \\
(\mathrm{n}=33)\end{array}$ & $\begin{array}{l}\text { International, } \\
\text { multicentre, } \\
\text { RDBPCT }\end{array}$ & $\begin{array}{l}\text { Primary: mean change in sputum } \\
\text { PA density }\left(\log _{10} \mathrm{CFU} \cdot \mathrm{g}^{-1}\right) \\
\text { from baseline to day } 28 \\
\text { Secondary: acute exacerbations, } \\
\text { PFT, quality of life scores, } \\
\text { safety and tolerability }\end{array}$ \\
\hline $\begin{array}{c}\text { ORRIOLS 1999, no } \\
\text { sponsor [32] }\end{array}$ & Spain & $\begin{array}{l}\text { Inclusion criteria: adult patients had bronchiectasis } \\
\text { diagnosed by bronchography, CT or both, had } \geqslant 3 \\
\text { positive sputum cultures for PA during the year prior to } \\
\text { study, and had been treated at least once with oral } \\
\text { ciprofloxacin in the past } 3 \text { months because of } \\
\text { exacerbation } \\
\text { Exclusion criteria: patients had CF, immunodeficiency, } \\
\text { enzymatic deficiency, congenital disease, } \\
\text { hypersensitivity or bacterial resistance to } \beta \text {-lactams or } \\
\text { aminoglycosides, or had kidney failure }\end{array}$ & $\begin{array}{c}\text { Tobramycin } \\
100 \mathrm{mg}+ \\
\text { Ceftazidime } \\
1000 \mathrm{mg} \text { via a jet } \\
\text { nebuliser, twice } \\
\text { daily, } 12 \text { months } \\
{[\mathrm{n}=8)}\end{array}$ & $\begin{array}{l}\text { Symptomatic } \\
\text { treatment with } \\
\text { oxygen, } \\
\text { bronchodilators } \\
\text { and } \\
\text { corticosteroids } \\
(n=9)\end{array}$ & $\begin{array}{l}\text { Randomised, } \\
\text { open-label, } \\
\text { controlled trial }\end{array}$ & $\begin{array}{l}\text { Microbiological study (bacterial } \\
\text { eradication) of sputum, } \\
\text { hospitalisation, use of } \\
\text { antibiotic, PFT, adverse } \\
\text { events, emergence of } \\
\text { bacterial resistance }\end{array}$ \\
\hline $\begin{array}{l}\text { SERISIER 2013, } \\
\text { sponsored by } \\
\text { Aradigm Corp. } \\
\text { [33] }\end{array}$ & $\begin{array}{c}\text { Australia, New } \\
\text { Zealand }\end{array}$ & $\begin{array}{l}\text { Inclusion criteria: adult patients had clinically stable } \\
\text { bronchiectasis diagnosed by CT, PA airway infection, } \\
\text { and had } \geqslant 2 \text { exacerbations requiring antibiotic therapy } \\
\text { in the preceding } 12 \text { months } \\
\text { Exclusion criteria: patients had CF, ABPA, or pulmonary } \\
\text { non-TB mycobacterial infection }\end{array}$ & $\begin{array}{l}\text { DRCFI lliposomal } \\
\text { ciprofloxacin } \\
150 \text { mg + free } \\
\text { ciprofloxacin } \\
60 \text { mgl via PARI } \\
\text { LC sprint } \\
\text { nebuliser, once } \\
\text { daily, three } \\
\text { treatment cycles } \\
\text { of } 28 \text { days "on" } \\
\text { inhaled therapy, } \\
28 \text { days "off" } \\
\text { (n=20) }\end{array}$ & $\begin{array}{l}\text { Matched placebo } \\
\text { (liposomal } 15 \mathrm{mg} \\
\text { or } 0.9 \% \text { saline) } \\
\text { via PARI LC } \\
\text { sprint nebuliser, } \\
\text { once daily, three } \\
\text { treatment cycles } \\
\text { of } 28 \text { days "on" } \\
\text { inhaled therapy, } \\
28 \text { days "off" } \\
\text { (n=22) }\end{array}$ & $\begin{array}{l}\text { International, } \\
\text { multicentre, } \\
\text { RDBPCT }\end{array}$ & $\begin{array}{l}\text { Primary: mean change in sputum } \\
\text { PA density }\left(\log _{10} \mathrm{CFU} \cdot \mathrm{g}^{-1} \text { ) }\right. \\
\text { from baseline to day } 28 \\
\text { Secondary: eradication of PA in } \\
\text { sputum, acute exacerbation, } \\
\text { PFT, } 6 \mathrm{MWT}, \mathrm{SGRQ} \text {, adverse } \\
\text { events and tolerability, } \\
\text { emergence of bacterial } \\
\text { resistance }\end{array}$ \\
\hline $\begin{array}{l}\text { TABERNERO 2014, } \\
\text { no sponsor } \\
\text { [28] }\end{array}$ & Spain & $\begin{array}{l}\text { Inclusion criteria: adult patients had non-CF } \\
\text { bronchiectasis diagnosed by HRCT, had chronic PA } \\
\text { airway infection after an acute exacerbation admission } \\
\text { and appropriate antimicrobial therapy } \\
\text { Exclusion criteria: not stated }\end{array}$ & $\begin{array}{l}\text { Colistin } \\
1 \text { million IU via a } \\
\text { nebuliser, twice } \\
\text { daily, } 1 \text { year } \\
(n=20)\end{array}$ & $\begin{array}{l}\text { Conventional } \\
\text { therapy, } 1 \text { year } \\
\qquad(n=19]\end{array}$ & $\begin{array}{l}\text { Randomised, } \\
\text { open-label, } \\
\text { controlled trial }\end{array}$ & $\begin{array}{l}\text { Primary: need for hospital } \\
\text { readmissions } \\
\text { Secondary: sputum microbiology, } \\
\text { clinical symptoms, PFT, } \\
\text { adverse events }\end{array}$ \\
\hline
\end{tabular}




\section{TABLE 1 Continued}

\begin{tabular}{|c|c|c|c|c|c|c|}
\hline Study & Country & Participants & Intervention & Control & Study design & Outcomes \\
\hline $\begin{array}{l}\text { TR02-107, 2009, } \\
\text { sponsored by } \\
\text { Insmed Inc. } \\
{[26,27]}\end{array}$ & $\begin{array}{c}\text { Bulgaria, } \\
\text { Greece, } \\
\text { Hungary, India, } \\
\text { Serbia, Ukraine }\end{array}$ & $\begin{array}{l}\text { Inclusion criteria: adult patients ( } \geqslant 18 \text { years) had non-CF } \\
\text { bronchiectasis diagnosed by } \mathrm{HRCT} \text {, had chronic PA } \\
\text { airway infection, had } \mathrm{SaO}_{2} \geqslant 90 \% \text { while breathing room } \\
\text { air, and were able to produce } \geqslant 0.5 \mathrm{~g} \text { sputum } \\
\text { Exclusion criteria: patients had CF, ABPA, aspiration of } \\
\text { foreign body, pulmonary TB or non-TB mycobacterial } \\
\text { infection, history of lung transplantation, } \mathrm{FEV}_{1}<50 \% \\
\text { predicated, use of any type of antibiotics within } 4 \text { weeks } \\
\text { prior to the study, evidence of biliary cirrhosis with } \\
\text { portal hypertension }\end{array}$ & $\begin{array}{c}\text { Arikace } \\
\text { (liposomal } \\
\text { amikacin) } 280 \mathrm{mg} \\
\text { or } 560 \mathrm{mg} \text {, via an } \\
\text { eFlow nebuliser, } \\
\text { once daily, } \\
28 \text { days ( } \mathrm{n}=44 \text { ) }\end{array}$ & $\begin{array}{c}\text { Matched } \\
\text { placebo } \\
\text { (liposomes in } \\
\text { 1.5\% saline) via } \\
\text { an eFlow } \\
\text { nebuliser, once } \\
\text { daily, } 28 \text { days } \\
(n=20)\end{array}$ & $\begin{array}{l}\text { International, } \\
\text { multicentre, } \\
\text { RDBPCT }\end{array}$ & $\begin{array}{l}\text { Primary: adverse events } \\
\text { Secondary: mean change in } \\
\text { sputum PA density, acute } \\
\text { exacerbation, hospitalisation, } \\
\text { PFT, SGRQ }\end{array}$ \\
\hline $\begin{array}{l}\text { WILSON 2013, } \\
\text { sponsored by } \\
\text { Bayer Pharma } \\
\text { AG [11] }\end{array}$ & $\begin{array}{c}\text { Australia, } \\
\text { Germany, Spain, } \\
\text { Sweden, UK, } \\
\text { USA }\end{array}$ & $\begin{array}{l}\text { Inclusion criteria: adult patients ( } \geqslant 18 \text { years) had non-CF } \\
\text { bronchiectasis diagnosed by HRCT, had } \geqslant 2 \\
\text { exacerbations requiring systemic antibiotics or } \geqslant 1 \\
\text { hospitalisation for intravenous antibiotics in the previous } \\
12 \text { months, had stable disease in the preceding } 30 \text { days, } \\
\text { and were able to produce sputum ( } \geqslant 5 \mathrm{~mL} \text { ) that was } \\
\text { culture positive for potential respiratory pathogens } \\
\text { Exclusion criteria: patients had active non-TB } \\
\text { mycobacterial infection, recent significant haemoptysis, } \\
\text { use of nebulised antibiotics as maintenance treatment, } \\
\text { or use of systemic antibiotics for exacerbation within } \\
4 \text { weeks before randomisation }\end{array}$ & $\begin{array}{l}\text { Ciprofloxacin } \\
32.5 \text { mg via dry } \\
\text { powder for } \\
\text { inhalation, twice } \\
\text { daily, } 28 \text { days } \\
(\mathrm{n}=60)\end{array}$ & $\begin{array}{l}\text { Matching } \\
\text { placebo via dry } \\
\text { powder for } \\
\text { inhalation, twice } \\
\text { daily, } 28 \text { days } \\
(n=64)\end{array}$ & $\begin{array}{l}\text { International } \\
\text { multicentre, } \\
\text { RDBPCT }\end{array}$ & $\begin{array}{l}\text { Primary: mean change in sputum } \\
\left.\text { bacterial load (log }{ }_{10} \mathrm{CFU} \cdot \mathrm{g}^{-1}\right) \\
\text { from baseline to day } 28 \\
\text { Secondary: acute exacerbation, } \\
\text { hospitalisation, sputum } \\
\text { analysis (bacterial eradication, } \\
\text { volume, colour), PFT, markers } \\
\text { of systemic inflammation, } \\
\text { SGRQ, adverse events, } \\
\text { emergence of bacterial } \\
\text { resistance }\end{array}$ \\
\hline
\end{tabular}

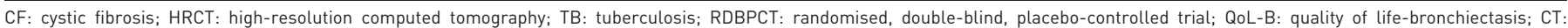

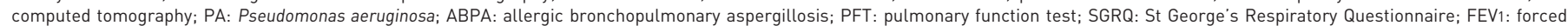

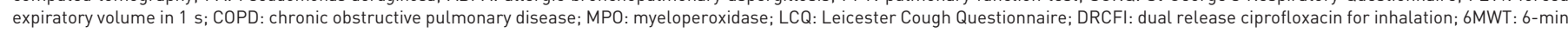
walk test; $\mathrm{SaO}_{2}$ : arterial oxygen saturation.

95\% CI -2.07--0.73; ciprofloxacin, two trials with 122 patients, WMD -3.52, 95\% CI -4.64--2.40; Chi-squared $=10.14$, degrees of freedom $=2, p=0.006$ ), but not between different bacteria ( $P$. aeruginosa, four trials with 217 patients, WMD -2.48, 95\% CI -4.46--0.51; any bacterial pathogen, one trial with 85 patients, WMD -3.35, 95\% CI -4.61--2.09; Chi-squared $=0.53$, degrees of freedom $=1, \mathrm{p}=0.47$ ). Among two trials on 160 patients, which were not included in the meta-analysis, one trial on 65 patients [31] showed a lower median (IQR) sputum bacterial load in the gentamicin group compared with the placebo group at the end of the 12-month treatment (2.96 (1.0-5.9) versus 7.67 (7.34-8.17) $\log _{10} \mathrm{CFU} \cdot \mathrm{g}^{-1}$, $\mathrm{p}<0.0001)$. Another unpublished trial $[24,25]$ on 95 patients also reported a greater reduction in sputum $P$. aeruginosa density from baseline to the end of 28-day treatment in both ciprofloxacin $100 \mathrm{mg}$ and $200 \mathrm{mg}$ groups compared to the placebo group $\left(-3.84,-2.94\right.$ and $0.437 \log _{10} \mathrm{CFU} \cdot \mathrm{g}^{-1}$, respectively; $\left.\mathrm{p}<0.001\right)$.

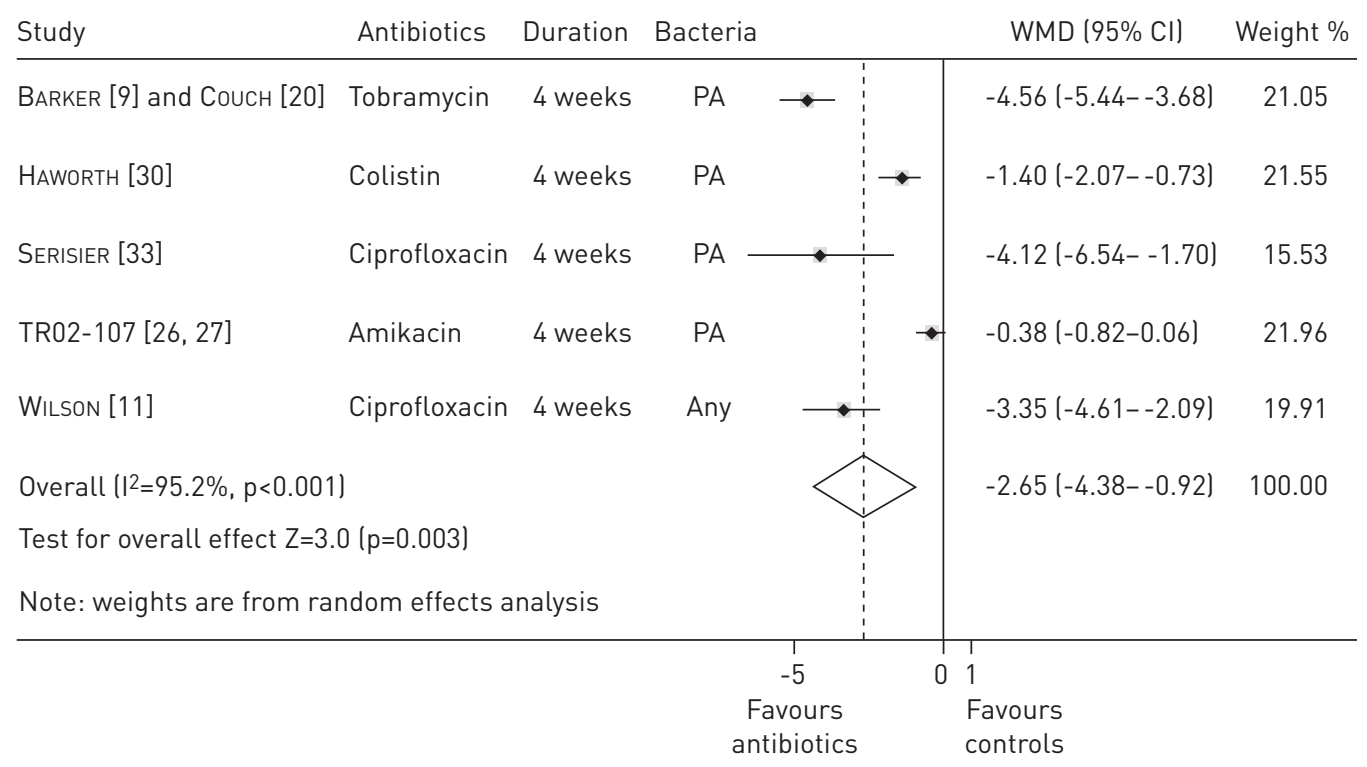

FIGURE 2 Effects of inhaled antibiotics on reduction of sputum bacterial load $\left(\log _{10} \mathrm{CFU} \cdot \mathrm{g}^{-1}\right)$. WMD: weighted mean difference; PA: Pseudomonas aeruginosa. 


\begin{tabular}{|c|c|c|c|c|c|c|c|c|c|}
\hline Study & Antibiotics & Duration & Bacteria & Intervention & Control & & & $\begin{array}{l}\text { Risk ratio } \\
(95 \% \mathrm{CI})\end{array}$ & $\begin{array}{c}\text { Weight } \\
\%\end{array}$ \\
\hline BARKER [9] and COUCH [20] & Tobramycin & 4 weeks & PA & $13 / 37$ & $0 / 35$ & & $\longrightarrow$ & $25.58(1.58-414.63)$ & 8.69 \\
\hline DROBNIC [29] & Tobramycin & 6 months & PA & $4 / 20$ & $4 / 20$ & & - & $1.00(0.29-3.45)$ & 22.33 \\
\hline ORRIOLS [32] & $\begin{array}{l}\text { Ceftazidime + } \\
\text { tobramycin }\end{array}$ & 12 months & PA & $0 / 7$ & $0 / 8$ & & 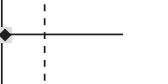 & $1.13(0.03-50.41)$ & 5.24 \\
\hline SERISIER [33] & Ciprofloxacin & 4 weeks & PA & $12 / 20$ & $3 / 22$ & & $\longrightarrow$ & $4.40(1.45-13.36)$ & 24.18 \\
\hline WILSON [11] & Ciprofloxacin & 4 weeks & Any & $14 / 40$ & $4 / 49$ & & & $4.29(1.53-12.01)$ & 25.38 \\
\hline \multicolumn{10}{|c|}{ Test for overall effect $Z=2.97(p=0.003$ ) } \\
\hline \multicolumn{10}{|c|}{ Note: weights are from random effects analysis } \\
\hline & & & & & & $\begin{array}{r}0.51 \\
\text { Favours } \\
\text { controls }\end{array}$ & $\begin{array}{cc}10 \\
\text { Favours } \\
\text { antibiotics }\end{array}$ & & \\
\hline
\end{tabular}

FIGURE 3 Effects of inhaled antibiotics on bacterial eradication from sputum. PA: Pseudomonas aeruginosa.

Six trials $[9,11,29,31-33]$ compared bacterial eradication rate between the inhaled antibiotic group and the control group. The meta-analysis of six trials with a total of 315 patients showed that inhaled antibiotics were associated with a four-fold higher chance of achieving bacterial eradication from sputum (risk ratio 4.2, 95\% CI 1.62-10.64; $\mathrm{p}=0.003, \mathrm{I}^{2}=51 \%$ ) (fig. 3). NNT was three. Subgroup analysis showed no statistically significant difference in achieving bacterial eradication between types of antibiotics (aminoglycosides versus ciprofloxacin: Chi-squared $=0.05$, degrees of freedom $=1 ; \mathrm{p}=0.82$ ) and types of bacteria in sputum ( $P$. aeruginosa versus any bacterial pathogen: Chi-squared $=0.55$, degrees of freedom $=1$; $\mathrm{p}=0.46)$.

\section{Clinical outcomes}

Five trials $[11,26,27,30,31,33]$ reported the number of patients experiencing at least one acute exacerbation. The meta-analysis of five trials with a total of 406 patients showed that inhaled antibiotics significantly reduced the risk of acute exacerbations compared with controls (risk ratio 0.72, 95\% CI $0.55-0.94 ; \mathrm{p}=0.02, \mathrm{I}^{2}=34.7 \%$ ) (online supplementary fig. S1). NNT was five. Four trials [21-23, 29-31] compared time to first exacerbation between treatment groups, but we obtained the data from only two of these trials. One trial [30] on 144 patients used time to first exacerbation as the primary efficacy outcome. ITT analysis did not show a statistically significant difference in median time to first exacerbation between the colistin and placebo groups (165 versus 111 days, $\mathrm{p}=0.11$ ); however, the secondary analysis in patients taking $\geqslant 81 \%$ of prescribed doses $(n=106)$ yielded a positive result ( 168 versus 103 days, $\mathrm{p}=0.038)$. In another trial [31] on 65 patients, median (IQR) time to first exacerbation was 120 (87-161.5) days in the gentamicin group compared with $61.5(20.7-122.7)$ days in the placebo group $(\mathrm{p}=0.02)$. One trial on 60 patients [29] reported the number of exacerbations per patient during a 6-month treatment period and did not find a statistically significant difference between the tobramycin and placebo groups.

Four trials $[9,11,26,27,32]$ reported the effects of inhaled antibiotics on reduction of risk of unscheduled hospitalisations. The meta-analysis of four trials with a total of 275 patients did not show a statistically significant difference in the incidence of hospitalisation between the antibiotic group (nine out of 147, $6.1 \%$ ) and the control group (14 out of $128,10.9 \%$ ) (risk ratio $0.59,95 \%$ CI $0.14-2.51, \mathrm{p}=0.48$ ). NNT was 21 . Two trials $[29,32]$ compared the number of hospitalisations and number of days spent in hospital per patient between the antibiotic and control groups. The meta-analysis of these two trials with a total of 77 patients showed that inhaled antibiotics significantly reduced the number of hospitalisations per patient 
(WMD $-0.61,95 \%$ CI $-1.04--0.18 ; \mathrm{p}=0.006$ ) and the number of days spent in hospital per patient (WMD -10.8 days, $95 \%$ CI $-18.8--2.81$ days; $\mathrm{p}=0.008)$.

One trial with 74 patients [9] reported clinical response to treatment, showing that $62 \%$ of patients treated with nebulised tobramycin for 4 weeks had investigator-assessed clinical improvement compared with $38 \%$ of placebo patients (OR 2.7, 95\% CI 1.1-6.9).

\section{Markers of airways and systemic inflammation}

One trial with 65 patients [31] reported sputum myeloperoxidase (MPO) and free elastase levels, and showed that patients treated with nebulised gentamicin for 12 months had significantly lower sputum MPO and free elastase levels compared with patients receiving $0.9 \%$ saline. Three trials $[11,29,31]$ reported markers of systemic inflammation such as C-reactive protein and erythrocyte sedimentation rate, but the data were not suitable for meta-analysis. None of these trials found a statistically significant difference between the inhaled antibiotic and control groups.

\section{Pulmonary function tests}

Nine trials $[9,11,24-27,29-33]$ reported mean change from baseline in forced expiratory volume in $1 \mathrm{~s}$ (FEV1) \% predicted and we obtained suitable data from all but one of these trials [24, 25] for pooling of the results. The meta-analysis of eight trials with 558 patients showed a small, but statistically significant, difference in mean change in $\mathrm{FEV}_{1} \%$ pred in favour of the control group (WMD $-0.66 \%, 95 \% \mathrm{CI}$ $\left.-1.13--0.29 \% ; \mathrm{p}=0.005, \mathrm{I}^{2}=90.7 \%\right)$.

\section{Health-related quality of life scores}

Nine trials [11,21-27, 29-31, 33] reported health-related quality of life scores. Five trials [11, 26, 27, 29-31] used the St George's Respiratory Questionnaire (SGRQ), which consists of 76 items rated on a scale 0-100, divided into three domains: respiratory symptoms, activities, and social and psychological impact. A higher score indicates a poorer quality of life. One trial [33] used both the SGRQ and Leicester Cough Questionnaire (LCQ), which is a 19-item self-completed quality of life measure of chronic cough validated for use in bronchiectasis. Two trials [21-23] used a new, disease-specific tool, the Quality of LifeBronchiectasis (QoL-B). The data from five trials with a total of 407 patients that used SGRQ were suitable for the meta-analysis, showing no statistically significant difference between the inhaled antibiotic and control groups in terms of improvement in SGRQ scores (WMD -1.49, 95\% CI -5.79-2.80; $\mathrm{p}=0.50$, $\mathrm{I}^{2}=75.8 \%$ ). In contrast, one trial with 65 patients [31] showed that $87.5 \%$ of patients treated with nebulised gentamicin for 12 months had an improvement in SGRQ scores of $\geqslant 4$ compared with $19.2 \%$ in the placebo group $(p<0.004)$. Similar results were obtained when the LCQ was used for measuring quality of life. Two trials [21-23] used mean change in QoL-B from baseline to day 28 as the primary efficacy outcome. One trial (AIR-BX2), but not the other (AIR-BX1), showed that patients treated with aztreonam had a statistically significant improvement in QoL-B $(\mathrm{p}=0.011)$ compared with the placebo group, although the magnitude of change was less than the predefined minimal important difference (online supplementary table S1).

\section{Safety of inhaled antibiotics}

The safety outcomes included adverse events, drug toxicity, tolerability and emergence of bacterial resistance. Eight trials $[9,11,26,27,29-33]$ contributed data to the meta-analysis of at least one safety outcome. The safety data of four unpublished trials $[21-25,28]$ are summarised in online supplementary table S1.

\section{Adverse events}

Table 2 shows the comparison of adverse events between the inhaled antibiotic and control groups. A total of 11 patients died during the treatment period, of whom seven were from the inhaled antibiotic group and four from the control group (eight trials with 590 patients; risk ratio $1.28,95 \%$ CI $0.44-3.71 ; \mathrm{p}=0.65$, $\mathrm{I}^{2}=0 \%$ ). One trial with 60 patients [29] reported five deaths due to respiratory failure: four between 2 and 3 months of tobramycin treatment, and one in the second month of the placebo period. These patients had worse baseline pulmonary function compared with the remaining surviving patients. One trial on 144 patients [30] reported three deaths (one from the colistin group and two from the placebo group), which were all considered unlikely to be related to the study drug. Another trial with 65 patients [31] reported two deaths from the gentamicin group: one patient died from previously undiagnosed metastatic colorectal cancer at 3 months and another died from myocardial infarction at 5 months. The most common adverse event was bronchospasm, which occurred in $10 \%$ of patients treated with inhaled antibiotics and $2.3 \%$ in the control group (seven trials with 526 patients; risk ratio 2.96, 95\% CI 1.30-6.73; $\mathrm{p}=0.01, \mathrm{I}^{2}=0 \%$ ). NNH was 13 . The subgroup analysis showed that inhaled aminoglycosides significantly increased the risk of 
TABLE 2 Adverse events of inhaled antibiotics in patients with non-cystic fibrosis bronchiectasis

Adverse events

Death
Withdrawal due to
$\quad$ adverse events
Bronchospasm
Cough
Haemoptysis

Events/patients
Trials n p-value

$I^{2} \%$

\section{Antibiotic group Control group}

$\begin{array}{cc}7 / 304(2.3) & 4 / 286 \\ 37 / 304(12.4) \\ & 35 / 286(12.2) \\ 26 / 260(10.0) & 6 / 266(2.3) \\ 15 / 97(15.5) & 14 / 101(13.9) \\ 6 / 97(6.2) & 5 / 101(4.9)\end{array}$

\author{
$1.28(0.44-3.71)$ \\ $1.00(0.67-1.50)$ \\ $2.96(1.30-6.73)$ \\ $0.54(0.03-0.52)$ \\ $1.26(0.39-4.01)$
}

$\begin{array}{cc}0.65 & 0 \\ 0.99 & 0 \\ & \\ 0.01 & 0 \\ 0.69 & 76.3 \\ 0.70 & 0\end{array}$

Data are presented as $\mathrm{n} / \mathrm{N}(\%)$, unless otherwise stated.

bronchospasm (four trials with 216 patients; risk ratio 4.78, 95\% CI 1.55-14.76, $\mathrm{p}=0.007$; NNH 7), while inhaled ciprofloxacin and colistin were not significantly associated with the occurrence of this adverse event (ciprofloxacin, two trials with 166 patients, risk ratio 1.07, 95\% CI 0.25-4.56, p=0.93; colistin, one trial with 144 patients, risk ratio 4.86, 95\% CI $0.58-40.59, \mathrm{p}=0.14$ ). There were no significant differences between the inhaled antibiotic and control groups in terms of withdrawal rate due to adverse events and other respiratory complaints such as cough and haemoptysis (table 2). All but one [26, 27] of the five trials $[9,26,27,29,31,32]$ using inhaled aminoglycosides reported nephrotoxic and/or ototoxic effects of these drugs, and none of them detected any significant toxicity. One [11] of the three trials [11, 24, 25, 33] using ciprofloxacin reported liver and renal function tests and found no significant changes.

\section{Emergence of bacterial resistance}

Seven trials $[9,11,29-33]$ reported the rate of emergence of bacterial resistance (number of isolates classified as resistant to the antibiotics/total number of isolates from sputum samples) in the inhaled antibiotic and control groups, but different methods were used for antibiotic susceptibility testing. The meta-analysis of seven trials with a total of 445 patients did not show a statistically significant difference in the incidence of emergence of bacterial resistance between the inhaled antibiotic group (17/217, 7.8\%) and the control group $\left(8 / 228,3.5 \%\right.$ ) (risk ratio $1.68,95 \%$ CI $0.62-4.52 ; \mathrm{p}=0.31, \mathrm{I}^{2}=15.7 \%$ ) (online supplementary fig. S2).

\section{Discussion}

This systematic review of 12 randomised trials involving 1264 participants and meta-analysis of eight trials with 590 participants shows that in adult patients with clinically stable non-CF bronchiectasis and chronic bronchial infection, inhaled antibiotics are more effective than placebo or symptomatic treatment in reducing sputum bacterial load, eradicating the bacteria from sputum and reducing the risk of acute exacerbations. However, this review did not find a significant benefit of inhaled antibiotics in reducing the risk of unscheduled hospitalisations or in improving health-related quality of life. Moreover, use of inhaled antibiotics is associated with a small, but statistically significant, reduction in FEV1\% pred.

The rationale for using inhaled antibiotics is the same as that for other inhaled therapies in many other lung diseases, that is, to deliver a relatively high dose of drugs directly to the site of disease with a reduced systemic absorption and toxicity [34]. Inhaled antibiotics have been widely used in CF patients since the early 1990s. A recently updated Cochrane systematic review of 19 randomised trials with 1724 CF patients showed that use of inhaled antibiotics for $\geqslant 4$ weeks improved lung function and reduced exacerbation rate. However, microbiological efficacy of these drugs was not reported [35].

This systematic review shows that 4 -week treatment with inhaled antibiotics resulted in an approximately 1000 -fold $\left(2.65 \log _{10} \mathrm{CFU} \cdot \mathrm{g}^{-1}\right)$ reduction in sputum bacterial load compared with placebo in adult patients with stable non-CF bronchiectasis and chronic bronchial infection. Moreover, inhaled antibiotics are four times more likely than placebo or symptomatic treatment to eradicate the bacteria from sputum in these patients. The NNT is three, which means for every three patients receiving inhaled antibiotics instead of placebo or symptomatic treatment, one additional patient will achieve bacterial eradication. Subgroup analysis shows that ciprofloxacin is more effective than aminoglycosides and colistin in reducing sputum bacterial density, but there is no statistically significant difference between different antibiotics in bacterial eradication rate. Subgroup analysis shows similar microbiological efficacy of inhaled antibiotics against $P$. aeruginosa and other bacterial pathogens. A significant reduction in sputum bacterial load was observed 
within a few days after treatment with inhaled antibiotics $[9,11,19]$; however, bacterial regrowth occurred and sputum bacterial density returned to baseline levels between 2 weeks and 3 months after cessation of treatment $[9,11,29,31,33]$.

This systematic review demonstrates some clinical benefits of inhaled antibiotics in adult patients with stable non-CF bronchiectasis. Inhaled antibiotics reduced the risk of having at least one acute exacerbation by $28 \%$. The NNT is five, which means that five patients need to be treated with inhaled antibiotics to prevent one additional exacerbation. Other potential clinical benefits of inhaled antibiotics include subjective clinical improvement, and reduction in number of hospitalisations and number of days spent in hospital per patient. However, this review did not find a statistically significant effect of inhaled antibiotics in reducing the risk of unscheduled hospitalisations and in improving health-related quality of life score. Moreover, use of inhaled antibiotics resulted in a small, but statistically significant, reduction in FEV1 \% pred. However, a reduction of $0.66 \%$ in FEV1 \% pred may not be clinically relevant.

This systematic review suggests that inhaled antibiotics have an acceptable safety profile. Bronchospasm was the most common adverse event, occurring in $10 \%$ of patients treated with inhaled antibiotics compared with $2.3 \%$ in the control group. Patients treated with inhaled aminoglycosides were almost five times more likely to have bronchospasm than those treated with placebo or symptomatic therapy with an NNH of seven, while inhaled ciprofloxacin and colistin did not significantly increase the risk of bronchospasm compared with the control group. Adverse events were generally tolerable because the withdrawal rate due to adverse events was the same in both the inhaled antibiotic and control groups. We found that patients in the inhaled antibiotic group had a statistically nonsignificant higher risk of death compared with the control group (risk ratio $1.28,95 \%$ CI $0.44-3.71$ ); however, there seems to be no causal relationship between 11 deaths and the use of inhaled antibiotics. Five (45.5\%) out of 11 deaths occurred in one trial between 2 and 3 months of treatment [29], and all five patients who died from respiratory failure had lower baseline pulmonary function. No significant nephrotoxic and/or ototoxic effects related to use of inhaled aminoglycosides were observed in the included trials.

Emergence of bacterial resistance is a major concern for any antibiotic therapy, particularly when used as a maintenance treatment. In 445 patients from seven trials, the incidence of emergence of bacterial resistance was $7.8 \%$ in the inhaled antibiotic group compared with 3.5\% in the control group, but this difference was not statistically significant.

Limitations should be considered when interpreting the results of this systematic review. High and unbalanced withdrawal rates in the treatment groups and lack of information on random sequence generation and allocation concealment in all but one of the 12 trials raise concerns about potential selection bias and attributions bias. However, the small number of trials with low risk of bias made it impossible to explore the impact of these potential biases on the results of the meta-analysis. This review may have insufficient power to detect the difference in the risk of unscheduled hospitalisations between treatment groups. The results of this review may not be extrapolated directly to paediatric patients because all 12 trials included in this review only involved adult patients. Non-CF bronchiectasis is a heterogeneous disorder with a variety of aetiologies, including immune deficiency, Mycobacterium tuberculosis or nontuberculous mycobacterial infections, hypersensitivity (allergic bronchopulmonary aspergillosis), aspiration of foreign bodies and systematic inflammatory conditions [36]. However, eight trials in this review excluded patients with one or more of the aforementioned conditions. The exclusion of such patients may limit the representativity of the study samples and, consequently, the generalisability of findings in this review. Publication bias is a major concern in all systematic reviews given that the trials reporting positive findings are more likely to be published [10,37]. A systematic review that only includes published studies may identify a spurious beneficial intervention effect [10]. In this review, we identified five unpublished trials, of which one contributed data to the meta-analysis. In the remaining four unpublished trials with 674 patients, only one used mean change from baseline in sputum bacterial load as the efficacy outcome and showed results similar to those identified by this review. Therefore, non-inclusion of four unpublished trials in the meta-analysis may not affect significantly the results of the primary efficacy outcome, mean change from baseline in sputum bacterial load. However, it is very likely that non-inclusion of these unpublished studies, especially three large trials [21-25], could substantially affect the results of the secondary outcomes such as acute exacerbations, changes in quality of life scores and safety end-points.

In conclusion, this systematic review suggests that in adult patients with clinically stable non-CF bronchiectasis and chronic bronchial infection, inhaled antibiotics may have microbiological and clinical benefits in reducing sputum bacterial load, eradicating bacteria from sputum and preventing acute exacerbations. Inhaled antibiotics may provide an effective suppressive antibiotic therapy with an acceptable safety profile in these patients. However, further multicentre randomised trials are still needed to better define the optimal regime and duration of treatment (long-term maintenance therapy or repeated short-term 
therapy), and to compare the effectiveness and safety of different inhaled antibiotics and between inhaled and systemic antibiotics. Further studies should include paediatric patients given that non-CF bronchiectasis remains one of the most important chronic lung diseases in the paediatric population, especially in developing countries.

\section{References}

Seitz AE, Olivier KN, Adjemian J, et al. Trends in bronchiectasis among Medicare beneficiaries in the United States, 2000 to 2007. Chest 2012; 142: 432-439.

Wang Z. Bronchiectasis: still a problem. Chin Med J 2014; 127: 157-172.

Seitz AE, Olivier KN, Steiner CA, et al. Trends and burden of bronchiectasis-associated hospitalisations in the United States, 1993-2006. Chest 2010; 138: 944-949.

Barker AF. Bronchiectasis. N Engl J Med 2002; 346: 1383-1393.

Cole PJ. Inflammation: a two-edged sword - the model of bronchiectasis. Eur J Respir Dis Suppl 1986; 147: 6-15. Chalmers JD, Smith MP, McHugh BJ, et al. Short- and long-term antibiotic treatment reduces airway and systemic inflammation in non-cystic fibrosis bronchiectasis. Am J Respir Crit Care Med 2012; 186: 657-665.

7 Evans DJ, Bara AI, Greenstone M. Prolonged antibiotics for purulent bronchiectasis in children and adults. Cochrane Database Syst Rev 2007; 2: CD001392.

8 Liberati A, Altman DG, Tetzlaff J, et al. The PRISMA statement for reporting systematic reviews and meta-analyses of studies that evaluate healthcare interventions: explanation and elaboration. BMJ 2009; 339: b2700.

9 Barker AF, Couch L, Fiel SB, et al. Tobramycin solution for inhalation reduces sputum Pseudomonas aeruginosa density in bronchiectasis. Am J Respir Crit Care Med 2000; 162: 481-485.

10 Higgins JPT, Green S, eds. Cochrane Handbook for Systematic Reviews of Interventions. Version 5.1.0. Oxford, The Cochrane Collaboration, 2011.

11 Wilson R, Welte T, Polverino E, et al. Ciprofloxacin dry powder for inhalation in non-cystic fibrosis bronchiectasis: a phase II randomised study. Eur Respir J 2013; 41: 1107-1115.

12 Antoniu S, Azoicai D. Ciprofloxacin DPI in non-cystic fibrosis bronchiectasis: a Phase II randomized study. Expert Opin Investig Drugs 2013; 22: 671-673.

13 Bilton D, Henig N, Morrissey B, et al. Addition of inhaled tobramycin to ciprofloxacin for acute exacerbations of Pseudomonas aeruginosa infection in adult bronchiectasis. Chest 2006; 130: 1503-1510.

14 Dhar R, Anwar GA, Bourke SC, et al. Efficacy of nebulised colomycin in patients with non-cystic fibrosis bronchiectasis colonised with Pseudomonas aeruginosa. Thorax 2010; 65: 553.

15 Crowther Labiris NR, Holbrook AM, Chrystyn H, et al. Dry powder versus intravenous and nebulised gentamicin in cystic fibrosis and bronchiectasis. A pilot study. Am J Respir Crit Care Med 1999; 160: 1711-1716.

16 de Lima LS, Bogossian M. Avaliacao da resposta clinica ao uso de antibioticos por via oral e via inalatoria em pacientes portadores de bronquiectasias [Clinical response to inhalation and oral antibiotics in patients with bronchiectasis]. Rev Assoc Med Bras 1999; 45: 229-236.

17 Scheinberg P, Shore E. A pilot study of the safety and efficacy of tobramycin solution for inhalation in patients with severe bronchiectasis. Chest 2005; 127: 1420-1426.

18 Steinfort DP, Steinfort C. Effect of long-term nebulised colistin on lung function and quality of life in patients with chronic bronchial sepsis. Intern Med J 2007; 37: 495-498.

19 Lin HC, Cheng HF, Wang CH, et al. Inhaled gentamicin reduces airway neutrophil activity and mucus secretion in bronchiectasis. Am J Respir Crit Care Med 1977; 155: 2024-2029.

20 Couch LA. Treatment with tobramycin solution for inhalation in bronchiectasis patients with Pseudomonas aeruginosa. Chest 2001; 120: Suppl., 114S-117S.

21 Gilead Sciences. Safety and effectiveness of AZLI (an inhaled antibiotic) in adults with non-cystic fibrosis bronchiectasis (AIR-BX1). http://clinicaltrials.gov/ct2/show/NCT01313624 Date last updated: March 7, 2014. Date last accessed: March 10, 2014

22 Gilead Sciences. Safety and effectiveness of AZLI (an inhaled antibiotic) in adults with non-cystic fibrosis bronchiectasis (AIR-BX2). Date last updated: March 7, 2014. Date last accessed: March 10, 2014.

23 Barker A, O’Donnell A, Thompson PJ, et al. Two phase 3 placebo-controlled trials of aztreonam lysine for inhalation (AZLI) for non-cystic fibrosis bronchiectasis (NCFB). Eur Respir J 2013; 42: Suppl. 57, 877s.

24 Aradigm Corp. Safety and efficacy study of ciprofloxacin for inhalation in patients with non-cystic fibrosis bronchiectasis "ORBIT-1". http://clinicaltrials.gov/show/NCT00889967 Date last updated: August 27, 2012. Date last accessed: March 10, 2014

25 Aradigm Reports Successful ORBIT-1 Bronchiectasis Study with Inhaled Liposomal Ciprofloxacin. Available from: www.drugs.com/clinical_trials/aradigm-reports-successful-orbit-1-bronchiectasis-study-inhaled-liposomal-ciprofloxacin11989.html Date last updated: June 2011. Date last accessed: March 10, 2014.

26 Insmed. A study to determine the safety and tolerability of Arikace ${ }^{\mathrm{TM}}$ versus placebo in patients who have bronchiectasis. http://clinicaltrials.gov/show/NCT00775138 Date last updated: May 31, 2012. Date last accessed: March 10, 2014.

27 Insmed, Inc. TR02-107 trial report. Available from: www.insmed.com/pdf/Pub9\%209_13_09.pdf Date last updated: September 13, 2009. Date last accessed: March 10, 2014.

28 Tabernero E, Gil P, Alkiza R, et al. Inhaled colistin in elderly patients with non-cystic fibrosis bronchiectasis and chronic Pseudomonas aeruginosa (PA) bronchial infection. Chest 2014; 145: 431A.

29 Drobnic ME, Suñé P, Montoro JB, et al. Inhaled tobramycin in non-fibrosis patients with bronchiectasis and chronic bronchial infection with Pseudomonas aeruginosa. Ann Pharmacother 2005; 39: 39-44.

30 Haworth CS, Foweraker JE, Wilkinson P, et al. Inhaled colistin in patients with bronchiectasis and chronic Pseudomonas aeruginosa infection. Am J Respir Crit Care Med 2014; 189: 975-982.

31 Murray MP, Govan JR, Doherty CJ, et al. A randomised controlled trial of nebulized gentamicin in non-cystic fibrosis bronchiectasis. Am J Respir Crit Care Med 2011; 183: 491-499.

32 Orriols R, Roig J, Ferrer J, et al. Inhaled antibiotic therapy in non-cystic fibrosis patients with bronchiectasis and chronic bronchial infection by Pseudomonas aeruginosa. Respir Med 1999; 93: 476-480. 
33 Serisier DJ, Bilton D, De Soyza A, et al. Inhaled, dual release liposomal ciprofloxacin in non-cystic fibrosis bronchiectasis (ORBIT-2): a randomised, double-blind, placebo-controlled trial. Thorax 2013; 68: 812-817.

34 Geller DE. Aerosol antibiotics in cystic fibrosis. Respir Care 2009; 54: 658-669.

35 Ryan G, Singh M, Dwan K. Inhaled antibiotics for long-term therapy in cystic fibrosis. Cochrane Database Syst Rev 2011; 3: CD001021.

36 Pasteur MC, Bilton D, Hill AT, et al. British Thoracic Society guideline for non-CF bronchiectasis. Thorax 2010; 65: i1-i58.

37 Hopewell S, Loudon K, Clarke MJ, et al. Publication bias in clinical trials due to statistical significance or direction of trial results. Cochrane Database Syst Rev 2009; 1: MR000006. 